\title{
Enhancement of co-seismic piezomagnetic signals near the edges of magnetization anomalies in the Earth's crust
}

\author{
Ken'ichi Yamazaki \\ Disaster Prevention Research Institute, Kyoto University, Gokasyo, Uji, Kyoto 611-0011, Japan \\ (Received February 10, 2010; Revised December 3, 2010; Accepted December 5, 2010; Online published February 28, 2011)
}

\begin{abstract}
A scheme is proposed for calculating the piezomagnetic fields that accompany the propagation of seismic waves through a non-uniformly magnetized crust. Examples of the calculations are provided. Generally, the calculation of the co-seismic piezomagnetic fields involves laborious three-dimensional volume integrals, even if the magnetization structure is two-dimensional. However, the calculation can be simplified by taking the Fourier transform of spatial distributions of the field into consideration. As an example, we have performed calculations for both the non-uniformly and uniformly magnetized crust with an intensity of $10 \mathrm{~A} / \mathrm{m}$. The incident seismic wave is considered to consist of Rayleigh waves with an amplitude of $5 \mathrm{~cm}$. The amplitudes of the piezomagnetic signals arising from uniformly magnetized crust are up to $0.2 \mathrm{nT}$, whereas those arising from non-uniformly magnetized crust are as large as $0.5 \mathrm{nT}$. This result indicates that the piezomagnetic field may be a plausible mechanism of generating co-seismic changes in the magnetic field with detectable amplitudes for large earthquakes, provided that the observation site is located near the magnetization boundaries.
\end{abstract}

Key words: Enhancement, piezomagnetic effect, magnetic anomalies, seismic waves, co-seismic variations, Fourier transforms.

\section{Introduction}

Variations in the geomagnetic field are frequently observed in association with the propagation of seismic waves (e.g., Eleman, 1965; Matsushima et al., 2002; Iyemori et al., 2005; Abdul Azeez et al., 2009). Although such variations are usually considered to arise from two mechanisms - the electrokinetic effect (Ishido and Mizutani, 1981; Pride, 1994; Garambois and Dietrich, 2001; Bordes et al., 2008) and electromagnetic induction due to ground motions (e.g., Iyemori et al., 1996; Honkura et al., 2002, 2004; Ujihara et al., 2004)—-there are other candidate mechanisms, such as those related to the resonance of ions (Honkura et al., 2009), the piezoelectric effect (Ogawa and Utada, 2000a, b) and the piezomagnetic effect. Among these other mechanisms, the piezomagnetic effect is investigated in the study reported here. The piezomagnetic effect involves changes in the magnetization of ferromagnetic minerals under mechanical stress (Stacey, 1964; Nagata, 1970; Stacey and Johnston, 1972). In the case that co-seismic variations are observed only in the magnetic field (e.g., Okubo et al., 2009), the contribution of the piezomagnetic effect must be taken into account because the electrokinetic effect and electromagnetic induction primarily generate electrical fields. Previous studies on variations in the geomagnetic field arising from the piezomagnetic effect have been performed in a framework of elastostatics (see the review by Sasai, 1994). To interpret co-

Copyright (C) The Society of Geomagnetism and Earth, Planetary and Space Sciences (SGEPSS); The Seismological Society of Japan; The Volcanological Society of Japan; The Geodetic Society of Japan; The Japanese Society for Planetary Sciences; TERRAPUB.

doi:10.5047/eps.2010.12.001 seismic variations in magnetic fields in terms of the piezomagnetic effect, however, theories should be reconsidered in a framework of elastodynamics.

An important finding of analyses of piezomagnetism based on elastostatics is the enhancement of the piezomagnetic field due to anomalies in the initial magnetization. $\mathrm{Nu}$ merical calculations performed under an assumption of synthetic two-dimensional (2-D) magnetization structures have revealed that the magnitudes of the piezomagnetic field are strongly enhanced near the edges of magnetic anomalies, thereby explaining observed variations in the magnetic field (Oshiman, 1990). An enhanced signal is also implicitly indicated by assuming a realistic 3-D magnetization structure (e.g., Nishida et al., 2007). By analogy with these findings, the amplitude of the piezomagnetic field accompanying the propagation of seismic waves is also expected to be enhanced near the edges of magnetization boundaries. Numerical investigations in elastodynamics are required to quantitatively estimate the amplitude of the expected piezomagnetic field. Simulations of co-seismic variations in the piezomagnetic field are additionally complicated by the fact that they involve 3-D volume integrals, even if the magnetization structure itself is two-dimensional.

In this paper, we propose a scheme for estimating piezomagnetic signals arising from seismic plain waves passing thorough non-uniform initial magnetizations that is based on minimal calculations. Numerical examples are presented to determine whether the piezomagnetic effect generates variations in the magnetic field during the propagation of seismic waves with detectable magnitudes. 


\section{Definition of the Problem}

Variations in the magnetic field due to the piezomagnetic effect, referred to here as piezomagnetic signals, are determined in terms of the constitutive law of the piezomagnetic effect and a basic law of magnetostatics. Experimental studies have revealed that changes in magnetization due to the piezomagnetic effect are proportional to the applied stress (Stacey, 1964; Nagata, 1970), which has been summarized by Sasai (1980) in a simple constitutive law:

$$
\Delta J_{i}=\frac{3}{2} \beta \Delta T_{i j} J_{j},
$$

where $\Delta J_{i}$ represents the change in magnetization due to the piezomagnetic effect, $\beta$ is the stress sensitivity, $\Delta T_{i j}$ is the increment in the deviatoric stress tensor, and $J_{j}$ is the initial magnetization. In Eq. (1) and below, the Einstein summation convention is used.

For a given spatial distribution of $\Delta J_{j}$, changes in the magnetic field $\Delta B_{i}$ corresponding to $\Delta J_{i}$ are determined based on the magnetic Coulomb's law:

$$
\Delta B_{i}(\mathbf{x})=-\frac{\partial}{\partial x_{i}} \frac{\mu^{m}}{4 \pi} \iiint_{V} \Delta J_{j}(\mathbf{a}) \frac{\partial}{\partial a_{j}} \frac{1}{|\mathbf{x}-\mathbf{a}|} d \mathbf{a},
$$

where $\mathbf{x}$ represents the location of the observation point, $\mu^{m}$ is the magnetic permeability, and $V$ is the volume within which $\Delta J_{i} \neq 0$. Although Eq. (2) is a law of magnetostatics, it provides an accurate approximation of $\Delta B_{i}$ corresponding to time-varying $\Delta J_{j}$, provided that the time taken for light to propagate from any point in $V$ to $\mathbf{x}$ is negligibly small compared with the period of temporal variations in $\Delta J_{i}$ and provided that the attenuation of electromagnetic waves due to the induction effect in the Earth's crust is negligibly small. Because the integrand of Eq. (2) rapidly decays according to the distance between $\mathbf{x}$ and a, it is possible to ignore the contributions from distances greater than a given threshold value (i.e., several tens of kilometers); hence, the time scale of light propagation is in the order of $10^{-4} \mathrm{~s}$. The dominant frequencies of seismic waves produced by large earthquakes are $<10 \mathrm{~Hz}$, meaning that the periods of temporal variations in $\Delta J_{i}$ are smaller than $10^{-1} \mathrm{~s}$. Therefore, the former of the above conditions is satisfied. In contrast, the validity of the latter condition remains uncertain; however, provided that we consider seismic surface waves with periods of several tens of seconds in a relatively resistive crust (i.e., with a resistivity $>100 \Omega \cdot \mathrm{m}$ ), the induction effect is almost negligible. Indeed, the skin depth corresponding to electromagnetic waves with a period of $1 / 30 \mathrm{~s}$ in a conductor with a resistivity of $100 \Omega \cdot \mathrm{m}$ is approximately $30 \mathrm{~km}$, which is a sufficiently large distance for contributions from magnetic sources at a greater distance to be ignored. Hence, the following discussion is continued based on Eq. (2).

To calculate the piezomagnetic field, explicit forms of the stress field must be given. Below, it is assumed that the time dependence of all physical values is given by $\exp (-i \omega t)$, where $\omega$ and $t$ are frequency and time, respectively; consequently, the time dependency is not explicitly written below. Consider the displacement field, $\mathbf{U}=\left(U_{1}, U_{2}, U_{3}\right)$, represented by

$$
\mathbf{U}(\mathbf{a})=\mathbf{A}(\mathbf{k}) \exp (i \mathbf{k} \cdot \mathbf{a})
$$

where $\mathbf{A}=\left(A_{1}, A_{2}, A_{3}\right), \mathbf{a}=\left(a_{1}, a_{2}, a_{3}\right)$, and $\mathbf{k}=$ $\left(k_{1}, k_{2}, k_{3}\right)$ denote the polarization of seismic waves, an arbitrary point in real-space, and the wavenumber vector, respectively. The corresponding stress field is given by Hook's law and Eq. (3), and the deviatoric stress tensors are represented by

$$
\Delta T_{i j}(\mathbf{a})=\mu^{e} P_{i j}(\mathbf{k}) \exp (i \mathbf{k} \cdot \mathbf{a}),
$$

where $\mu^{e}$ is rigidity and $P_{i j}(\mathbf{k})$ is a function that depends only on $\mathbf{k}$ :

$$
P_{i j}(\mathbf{k})=i\left[-\delta_{i j} k_{k} A_{k}(\mathbf{k})+\frac{3}{2}\left(k_{i} A_{j}(\mathbf{k})+k_{j} A_{i}(\mathbf{k})\right)\right] .
$$

Substituting Eq. (4) into Eq. (1), and substituting this in turn into Eq. (2), we obtain the following expression of the piezomagnetic signals:

$$
\Delta B_{i}(\mathbf{x})=\frac{1}{4 \pi} \beta \mu^{m} \mu^{e} C_{i, j}(\mathbf{x}, \mathbf{k}) P_{j k}(\mathbf{k}) J_{k},
$$

where $C_{i, j}$ is a function of

$$
C_{i, j}(\mathbf{x}, \mathbf{k})=\iiint_{V} \frac{\partial}{\partial x_{i}} \frac{\partial}{\partial x_{j}} \frac{1}{|\mathbf{x}-\mathbf{a}|} \exp (i \mathbf{k} \cdot \mathbf{a}) d \mathbf{a} .
$$

\section{Solution of the Piezomagnetic Field 3.1 Fourier transforms}

To obtain the solution of the piezomagnetic field, it is necessary to calculate the integral in Eq. (7). Although this is a difficult task, it is generally the case that the calculation of the magnetic field arising from sources in the Earth's crust becomes easier when considered in the frequency domain (e.g. Yamazaki, 2009). Hence, instead of calculating the integral directly, let us consider the double Fourier transforms of the integral with respect to $x_{1}$ and $x_{2}$ :

$$
\Delta B_{i}^{* *}\left(l_{1}, l_{2}, x_{3}\right)=\frac{1}{4 \pi} \beta \mu^{m} \mu^{e} C_{i, j}^{* *}(\mathbf{x}, \mathbf{k}) P_{j k}(\mathbf{k}) J_{k},
$$

where

$$
\begin{aligned}
C_{i, j}^{* *}\left(l_{1}, l_{2}, x_{3}, \mathbf{k}\right)= & \frac{1}{(2 \pi)^{2}} \int_{-\infty}^{+\infty} d x_{1} \int_{-\infty}^{+\infty} d x_{2} \\
& \cdot C_{i, j}(\mathbf{x}, \mathbf{k}) \exp \left[-i\left(l_{1} x_{1}+l_{2} x_{2}\right)\right] .
\end{aligned}
$$

The double asterisk represents the double Fourier transform of functions. In the case of $l_{1}=l_{2}=0$, the integral of Eq. (9) is zero for all $i$ and $j$, which is confirmed by exchanging the order of integration. In the case of $l_{1}^{2}+l_{2}^{2} \neq$ 0 , the integral is performed by utilizing a formula on the double Fourier transform (e.g., Sasai, 1991):

$$
\begin{aligned}
& \frac{1}{2 \pi} \int_{-\infty}^{+\infty} d r_{1} \int_{-\infty}^{+\infty} d r_{2} \cdot \frac{\exp \left[-i\left(l_{1} r_{1}+l_{2} r_{2}\right)\right]}{\sqrt{r_{1}^{2}+r_{2}^{2}+r_{3}^{2}}} \\
& =\frac{1}{l_{H}} \exp \left(-l_{H}\left|r_{3}\right|\right)
\end{aligned}
$$


where $l_{H}=\left(l_{1}^{2}+l_{2}^{2}\right)^{1 / 2}$. Using this formula together with a partial integration, $C_{i, j}^{* *}$ is represented by

$$
\begin{aligned}
& C_{i, j}^{* *}\left(l_{1}, l_{2}, x_{3}, \mathbf{k}\right) \\
& =\frac{1}{2 \pi} \cdot l_{H} \exp \left(+l_{H} x_{3}\right) \iiint_{V^{\prime}} d \mathbf{a} \cdot L_{i} L_{j} \\
& \times \exp \left\{i\left[\left(k_{1}-l_{1}\right) a_{1}+\left(k_{2}-l_{2}\right) a_{2}+\left(k_{3}+i l_{H}\right) a_{3}\right]\right\},
\end{aligned}
$$

where $\left(L_{1}, L_{2}, L_{3}\right)=\left(i l_{1} / l_{H}, i l_{2} / l_{H}, 1\right)$. The calculation of Eq. (11) is generally easier than that of Eq. (7) because the integral variables (i.e., $a_{1}, a_{2}$, and $a_{3}$ ) in Eq. (11) only appear in the exponential, whereas those in Eq. (7) also appear in the denominator.

\subsection{Explicit forms of the solutions}

First, let us consider an initial magnetization with a prism-shaped structure, as defined by

$$
V=\left\{\mathbf{a} \mid a_{1}^{l} \leq a_{1} \leq a_{1}^{u}, a_{2}^{l} \leq a_{2} \leq a_{2}^{u}, a_{3}^{l} \leq a_{3} \leq a_{3}^{u}\right\} .
$$

In this case, the integral is conducted separately with respect to each variable, yielding

$$
\begin{aligned}
C_{i, j}^{* *}\left(l_{1}, l_{2}, x_{3}, \mathbf{k}\right)= & \frac{1}{2 \pi} l_{H} L_{i} L_{j} \exp \left(l_{H} x_{3}\right) \\
& \times Q\left(k_{1}-l_{1} ; a_{1}^{l}, a_{1}^{u}\right) Q\left(k_{2}-l_{2} ; a_{2}^{l}, a_{2}^{u}\right) \\
& \times Q\left(k_{3}+i l_{H} ; a_{3}^{l}, a_{3}^{u}\right),
\end{aligned}
$$

where $Q$ denotes a three-variable function defined by

$$
\begin{aligned}
Q\left(p ; a^{l}, a^{u}\right) & =\int_{a^{l}}^{a^{u}} \exp (i p a) d a \\
& = \begin{cases}\frac{1}{i p}\left[\exp \left(i p a^{u}\right)-\exp \left(i p a^{l}\right)\right] & \text { for } p \neq 0, \\
a^{u}-a^{l} & \text { for } p=0 .\end{cases}
\end{aligned}
$$

Substituting Eq. (13) into Eq. (8) leads to the expression

$$
\begin{aligned}
\Delta B_{i}^{* *}\left(l_{1}, l_{2}, x_{3}\right)= & \frac{1}{8 \pi^{2}} \beta \mu^{m} \mu^{e} \exp \left(l_{H} x_{3}\right) l_{H} L_{i} L_{j} P_{j k} J_{k} \\
& \times Q\left(k_{1}-l_{1} ; a_{1}^{l}, a_{1}^{u}\right) Q\left(k_{2}-l_{2} ; a_{2}^{l}, a_{2}^{u}\right) \\
& \times Q\left(k_{3}+i l_{H} ; a_{3}^{l}, a_{3}^{u}\right) .
\end{aligned}
$$

Our purpose is to obtain an expression of the piezomagnetic field in real-space, which is derived by performing the reverse Fourier transform:

$$
\begin{aligned}
\Delta B_{i}\left(x_{1}, x_{2}, x_{3}\right)= & \int_{-\infty}^{+\infty} d l_{1} \int_{-\infty}^{+\infty} d l_{2} \\
& \cdot \Delta B_{i}^{* *}\left(l_{1}, l_{2}, x_{3}\right) \exp \left[i\left(l_{1} x_{1}+l_{2} x_{2}\right)\right] .
\end{aligned}
$$

Next, let us consider a 2-D magnetization structure defined by

$$
V=\left\{\mathbf{a} \mid-\infty \leq a_{1} \leq+\infty, a_{2}^{l} \leq a_{2} \leq a_{2}^{u}, a_{3}^{l} \leq a_{3} \leq a_{3}^{u}\right\} .
$$

Given that Eq. (17) is a special case of Eq. (12), the corresponding piezomagnetic field is determined by substituting Eq. (15) with $\left(a_{1}^{l}, a_{1}^{u}\right)$ into Eq. (16). Using a formula of the Fourier transform:

$$
\int_{-\infty}^{+\infty} \exp [i(k-l) a] d a=2 \pi \delta(k-l)
$$

where $\delta$ represents Dirac's Delta function (e.g., Arfken and Weber, 2000); the function $Q\left(k_{1}-l_{1},-\infty,+\infty\right)$ is replaced by $2 \pi \delta(k-l)$. The reverse Fourier transform of $\Delta B_{i}^{* *}$ with respect to the variable $l_{1}$ is thus accomplished, yielding

$$
\begin{aligned}
\Delta B_{i}^{*}\left(x_{1}, l_{2}, x_{3}\right)= & \frac{1}{4 \pi} \beta \mu^{m} \mu^{e} \exp \left(i k_{1} x_{1}\right) \exp \left(l_{H}^{\prime} x_{3}\right) \\
& \times l_{H}^{\prime} L_{i}^{\prime} L_{j}^{\prime} P_{j k} J_{k} \\
& \times Q\left(k_{2}-l_{2} ; a_{2}^{l}, a_{2}^{u}\right) \\
& \times Q\left(k_{3}+i l_{H}^{\prime} ; a_{3}^{l}, a_{3}^{u}\right)
\end{aligned}
$$

where $l_{H}^{\prime}=\left(k_{1}^{2}+l_{2}^{2}\right)^{1 / 2}$ and $\left(L_{1}^{\prime}, L_{2}^{\prime}, L_{3}^{\prime}\right)=$ $\left(i k_{1} / l_{H}^{\prime}, i l_{2} / l_{H}^{\prime}, 1\right)$. Note that the function $\Delta B_{i}^{*}$ also represents the Fourier transform of $\Delta B_{i}^{*}$, with respective to $x_{1}$ only. Therefore, $\Delta B_{i}$ is given by

$$
\Delta B_{i}\left(x_{1}, x_{2}, x_{3}\right)=\int_{-\infty}^{+\infty} \Delta B_{i}^{*}\left(x_{1}, l_{2}, x_{3}\right) \exp \left(i l_{2} x_{2}\right)
$$

Finally, consider the 1-D magnetization structure defined by

$V=\left\{\mathbf{a} \mid-\infty \leq a_{1} \leq+\infty,-\infty \leq a_{2} \leq+\infty, a_{3}^{l} \leq a_{3} \leq a_{3}^{u}\right\}$.

Given that this is also a special case of Eq. (12), the corresponding piezomagnetic field is calculated by substituting Eq. (15) into Eq. (16). Using Eq. (18) twice, the integration is finalized, and the expression of $\Delta B_{i}$ is obtained as follows:

$$
\begin{aligned}
\Delta B_{i}\left(x_{1}, x_{2}, x_{3}\right)= & \frac{1}{2} \beta \mu^{m} \mu^{e} \exp \left[i\left(k_{1} x_{1}+k_{2} x_{2}\right)\right] \\
& \times \exp \left(k_{H} x_{3}\right) k_{H} K_{i} K_{j} P_{j k} J_{k} \\
& \times Q\left(k_{3}+i k_{H} ; a_{3}^{l}, a_{3}^{u}\right),
\end{aligned}
$$

where $k_{H}=\left(k_{1}^{2}+k_{2}^{2}\right)^{1 / 2}$ and $\left(K_{1}, K_{2}, K_{3}\right)=$ $\left(i k_{1} / k_{H}, i k_{2} / k_{H}, 1\right)$.

In the cases of 2-D and 3-D structures, the integral of the reverse Fourier transform cannot generally be determined analytically. However, numerical calculations of these integrals are rapidly performed via the fast Fourier transform. Therefore, the use of these formulae has advantages over an approach involving the direct calculation of the volume integral using Eq. (7).

\section{Numerical Examples}

\subsection{Configuration of the problem}

Here, examples are presented of calculations of the piezomagnetic signals arising from the propagation of Rayleigh waves, using the formula obtained above. The 
(a) 2-D Structure
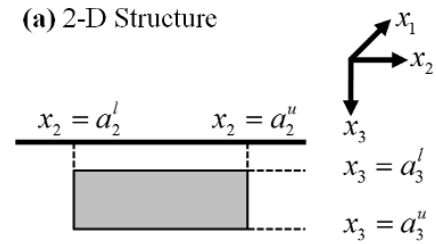

(b) 1-D Structure

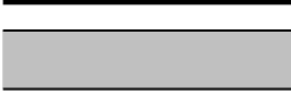

Fig. 1. Example of cross-sections representing the distribution of magnetization in the Earth's Crust. (a) Magnetization with a two-dimensional structure. (b) Magnetization with a one-dimensional structure. The $x_{1}$ axis is the direction in which the magnetic anomaly extends; the $x_{3}$ axis is vertically downward; the $x_{2}$ axis is chosen in such a way that $x_{1} x_{2} x_{3}$ forms a right-hand orthogonal system. The thick black lines represent the upper and the lower boundaries of the Earth's crust. Gray areas represent the magnetized crust.

displacement field of the Rayleigh wave is expressed by the sum of $\mathbf{U}_{1}$ and $\mathbf{U}_{2}$, each of which is represented in the form of Eq. (3), with

$$
\begin{gathered}
\mathbf{k}_{1}=k_{H} \times\left(\cos \theta, \sin \theta, i v_{R} \eta_{P}\right) \\
\mathbf{A}\left(\mathbf{k}_{1}\right)=A_{R} \times\left(i \cos \theta, i \sin \theta,-v_{R} \eta_{P}\right)
\end{gathered}
$$

and

$$
\begin{gathered}
\mathbf{k}_{2}=k_{H} \times\left(\cos \theta, \sin \theta, i v_{R} \eta_{S}\right) \\
\mathbf{A}\left(\mathbf{k}_{2}\right)=\frac{A_{R}}{2}\left(\frac{v_{R}^{2}}{v_{S}^{2}}-2\right) \times\left(i \cos \theta, i \sin \theta,-\frac{1}{v_{R} \eta_{S}}\right),
\end{gathered}
$$

where $\theta$ represents the angle between $x_{1}$ positive and the direction in which the Rayleigh wave is propagating; $A_{R}$ and $L_{R}$ represent the amplitude and wavenumber, respectively; $v_{P}, v_{S}$, and $v_{R}$ represent the velocities of the $P$-, $S$-, and Rayleigh waves, respectively; $\eta_{P}$ and $\eta_{S}$ represent $\left(v_{R}^{-2}-v_{P}^{-2}\right)^{1 / 2}$ and $\left(v_{R}^{-2}-v_{S}^{-2}\right)^{1 / 2}$, respectively (e.g., Lay and Wallace, 1995). Below, a Poisson solid is assumed, for which $v_{R} / v_{P}$ and $v_{S} / v_{P}$ take values of 0.531 and 0.577 , respectively. The piezomagnetic field that accompanies the Rayleigh wave is determined by the sum of the fields arising from $\mathbf{U}_{1}$ and $\mathbf{U}_{2}$.

To examine the degree of enhancement of the piezomagnetic field, two structures are considered with initial magnetizations expressed by $V$ in Eq. (21) and $V$ in Eq. (17). The former represents a horizontally uniform magnetization, whereas the latter represents a non-uniform magnetization. The intensity of the initial magnetization $(|\mathbf{J}|)$ and the thickness of the magnetization $\left(a_{3}^{l}\right.$ and $\left.a_{3}^{u}\right)$ are set to be the same in both cases. Cross-sections of the two cases are shown in Fig. 1.

In the formulation described in the previous section, the $x_{1} x_{2} x_{3}$-system (which is fixed to the geometry of the magnetization boundary) is employed; however, the magnetization boundary does not always coincide with the geographical directions (e.g., north-south and east-west). Therefore, it is more convenient to consider another right-hand orthogonal system, $X Y Z$, in which the $X$ and $Z$ directions correspond to magnetic north and vertically downward, respectively. Note that $Z$ is equivalent to $x_{3}$, but $X$ and $Y$ are not
Table 1. Parameters used in calculating changes in the magnetic field.

\begin{tabular}{lll}
\hline Parameter & Notation & Assumed value \\
\hline Upper and lower bounds & $a_{1}^{l}$ & $-50 \mathrm{~km}$ \\
of the magnetic anomalies (see Fig. 1) & $a_{1}^{u}$ & $+50 \mathrm{~km}$ \\
& $a_{3}^{l}$ & $0.5 \mathrm{~km}$ \\
& $a_{3}^{u}$ & $5.5 \mathrm{~km}$ \\
Height of observation & $x_{3}$ & $0 \mathrm{~m}$ \\
Intensity of initial magnetization & $|\mathbf{J}|$ & $10 \mathrm{~A} / \mathrm{m}$ \\
$P$-wave velocity & $v_{P}$ & $6.0 \mathrm{~km} / \mathrm{s}$ \\
Period of the Rayleigh wave & $T$ & $30 \mathrm{~s}$ \\
Amplitude of the Rayleigh wave & $A_{R}$ & $5 \times 10^{-2} \mathrm{~m}$ \\
Magnetic inclination & & $45^{\circ}$ \\
\hline
\end{tabular}

equivalent to $x_{1}$ and $x_{2}$, respectively. The direction of initial magnetization is assumed to be the same as that of the ambient geomagnetic field.

\subsection{Case studies}

For a case study, the set of parameters given in Table 1 is considered. The values of the depth and thickness of the magnetized layer and the intensity of magnetization were chosen based on a previous study in which they were estimated in a strongly magnetized area (Nishida et al., 2004). Figure 2 shows examples of the results corresponding to various combinations of the two directions that characterize the geometry of the problem: the direction in which the magnetization anomaly extends, measured from the $x_{1}$ axis $(D)$, and the direction in which the Rayleigh wave propagates, measured from the $x_{1}$ axis $(\theta)$. Calculations were performed for all combinations of $D=0^{\circ}, 45^{\circ}$, and $90^{\circ}$, and $\theta=D+0^{\circ}, D+45^{\circ}$, and $D+90^{\circ}$.

It is clear that for most of the calculated situations, the amplitudes of the piezomagnetic signals arising from the non-uniformly magnetized crust (referred to as $\Delta \mathbf{B}^{\mathrm{NU}}$ ) are much larger than those arising from the uniformly magnetized crust (referred to as $\Delta \mathbf{B}^{\mathrm{U}}$ ). For example, consider the results shown in the third row of the first column in Fig. 2, in which a Rayleigh wave propagates to the northeast over a north-south magnetic anomaly (i.e., $D=0^{\circ}$ and $\left.\theta=D+45^{\circ}\right)$. In this case, the $X, Y$, and $Z$ components of $\Delta \mathbf{B}^{\mathrm{U}}$ are $0.09,0.09$, and $0.13 \mathrm{nT}$, respectively. In contrast, those of $\Delta \mathbf{B}^{\mathrm{NU}}$ reach $0.10,0.47$, and $0.49 \mathrm{nT}$, respectively, at a point just above the contrast in magnetization. Given that the minimum distinguishable amplitude of co-seismic variations in the magnetic field is approximately $0.1 \mathrm{nT}$, signals in the $Y$ and $Z$ components are expected to be detectable in the case of non-uniform magnetization, whereas the detection of clear signals is expected to be difficult in the case of uniform magnetization.

The results just described were obtained for a specific geometry of the magnetized layer. To confirm that the significant enhancement of the piezomagnetic signal is a general feature in the case of the non-uniformly magnetized crust, $\mathbf{B}^{\mathrm{NU}}$ and $\mathbf{B}^{\mathrm{U}}$ are estimated for various depths and thicknesses of the magnetized layer. As an example, calculations are performed for the setting with $D=45^{\circ}$ and $\theta=0^{\circ}$; the values of the other parameters are those listed in Table 1, unless specified otherwise.

Figure 3 shows the maximum amplitudes of $\mathbf{B}^{\mathrm{NU}}$ (re- 

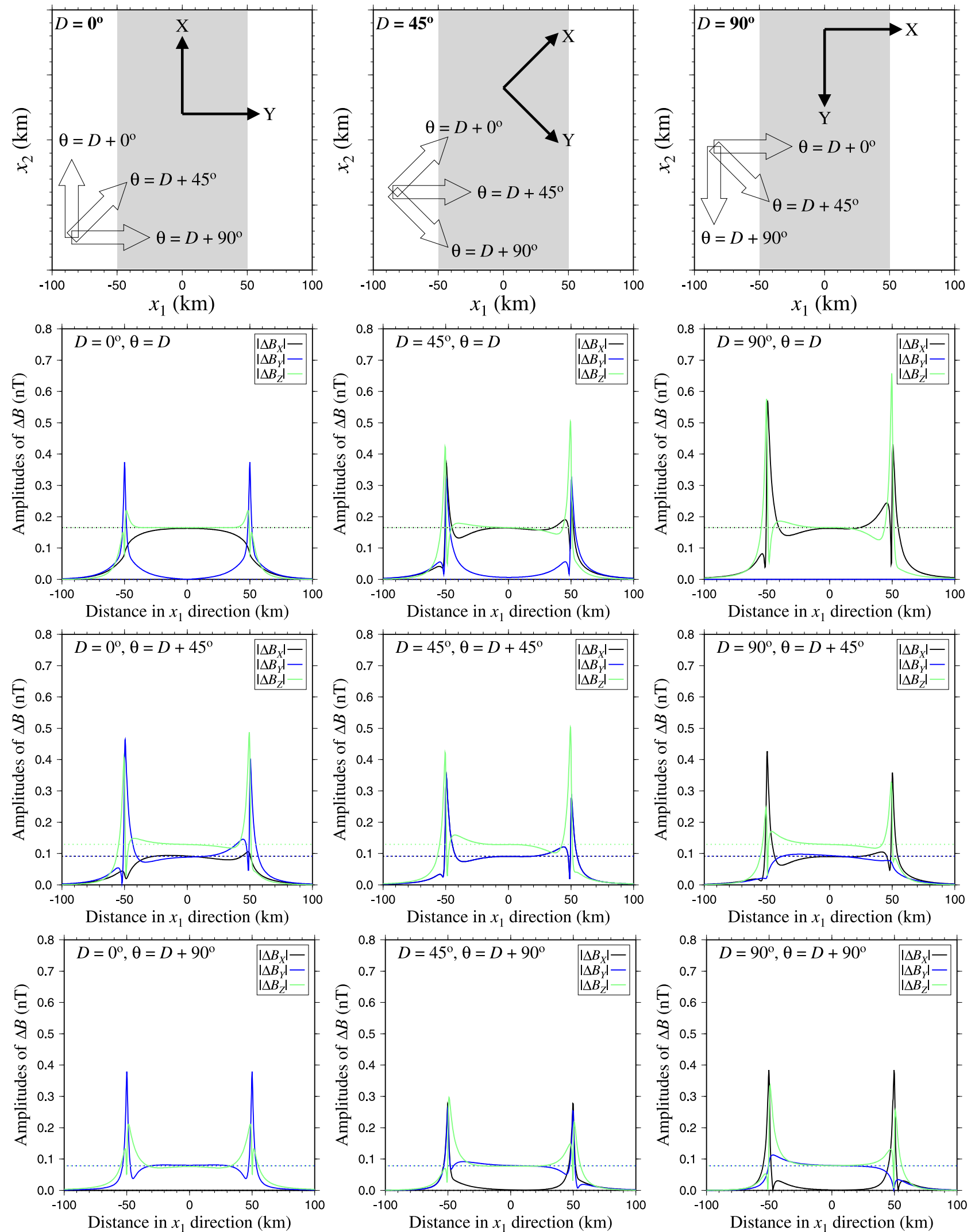

Fig. 2. Amplitudes of the piezomagnetic signals arising from Rayleigh waves propagating over magnetic anomalies. Panels in the top row show plan views of definitions of the directions employed in the calculation. Definitions of the $x_{1}$ and $x_{2}$ axis are provided in Fig. 1. The other panels show the corresponding results. Curves represent the amplitudes of signals arising from non-uniformly magnetized crust (i.e., Fig. 1(a)), and dashed lines represent those arising from uniformly magnetized crust (i.e., Fig. 1(b)). The second, third, and fourth rows from the top show results corresponding to Rayleigh waves propagating to the north, northeast, and east, respectively. The left, center, and right columns show plan views and results corresponding to the cases of magnetic anomalies oriented north-south, northwest-southeast, and east-west, respectively. In each panel, $D$ represents the direction of magnetic north (i.e., $X$ axis), as measured from the direction in which the magnetic anomaly extends (i.e., $x_{1}$ axis), and $\theta$ represents the direction in which the Rayleigh wave propagates, as measured from magnetic north. 
(a)

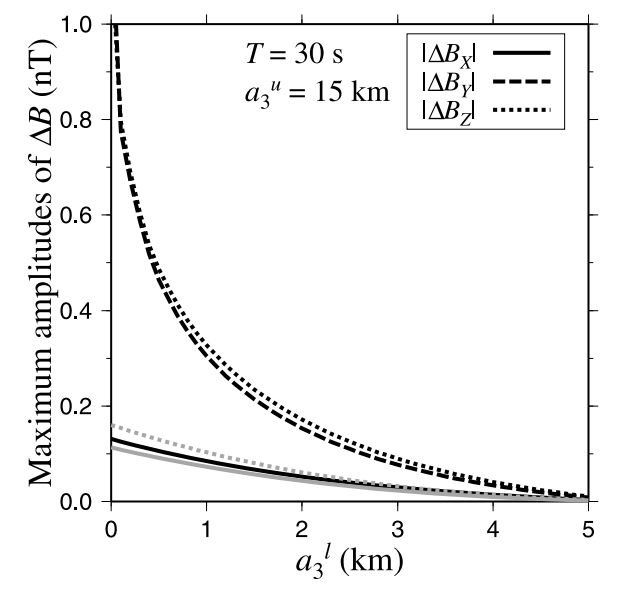

(b)

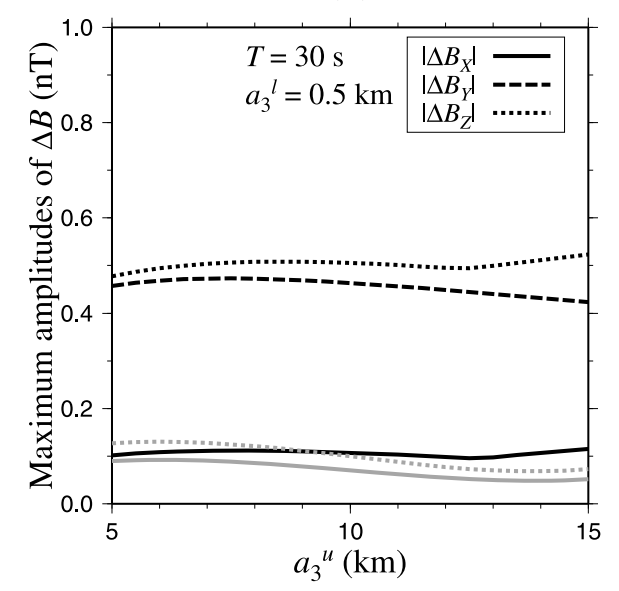

(c)

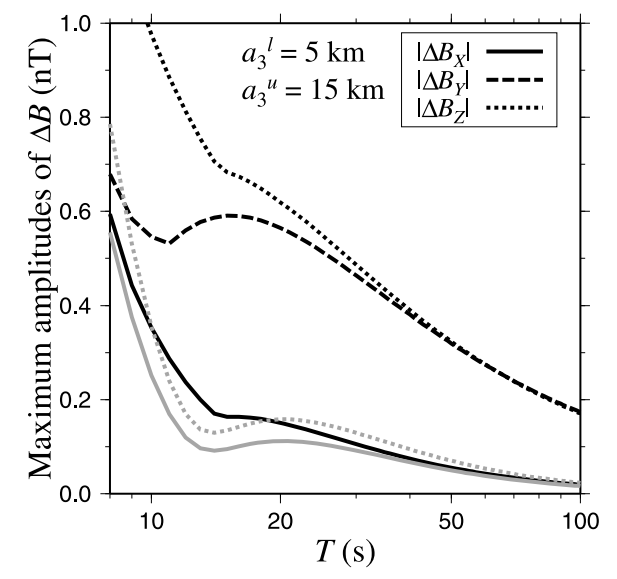

Fig. 3. Maximum amplitudes of the expected piezomagnetic signals ( $\Delta B_{X}, \Delta B_{Y}$, and $\Delta B_{Z}$ ) plotted against assumed parameters. (a) Dependency on $a_{3}^{l}$, i.e., depth of the top of the magnetized layer. (b) Dependency on $a_{3}^{u}$, i.e., depth of the bottom boundary of the magnetized layer. (c) Dependency of $T$, i.e., period of the Rayleigh wave. Values of unspecified parameters are set to $a_{3}^{l}=0.5(\mathrm{~km}), a_{3}^{u}=5.5$ $(\mathrm{km}), T=30 \mathrm{~s}$, and those listed in Table 1 . Solid, dashed, and dotted curves represent $\Delta B_{X}, \Delta B_{Y}$, and $\Delta B_{Z}$, in the case of magnetization, respectively. Curves in black and gray represent results corresponding to the non-uniformly and uniformly magnetized crust, respectively (i.e., $\left(\mathbf{B}^{\mathrm{NU}}\right)_{\mathrm{MAX}}$ and $\mathbf{B}^{\mathrm{U}}$, respectively). The values of $\Delta B_{X}$ and $\Delta B_{Y}$, in the latter case are the same, so that only $\Delta B_{X}$ is plotted. ferred to as $\left.\left(\mathbf{B}^{\mathrm{NU}}\right)_{\mathrm{MAX}}\right)$ near the edges of the magnetization boundary and $\mathbf{B}^{\mathrm{U}}$. The first step is to examine how the expected amplitude of the piezomagnetic signals changes in relation to the depth of the top of the magnetized layer $\left(a_{3}^{l}\right)$. An important result shown in Fig. 3(a) is that the factor of amplification (i.e., the ratio between $\left(\mathbf{B}^{\mathrm{NU}}\right)_{\text {MAX }}$ and $\mathbf{B}^{\mathrm{U}}$ ) remains largely unchanged as $\left(\mathbf{B}^{\mathrm{NU}}\right)_{\text {MAX }}$ and $\mathbf{B}^{\mathrm{U}}$ decrease with increases in $a_{3}^{l}$. The next step is to examine how $\left(\mathbf{B}^{\mathrm{NU}}\right)_{\mathrm{MAX}}$ and $\mathbf{B}^{\mathrm{U}}$ change in relation to the bottom depth of the magnetized layer $\left(a_{3}^{l}\right)$ (Fig. 3(b)). The results show that $\left(\mathbf{B}^{\mathrm{NU}}\right)_{\text {MAX }}$ and $\mathbf{B}^{\mathrm{U}}$ are almost constant with increasing $a_{3}^{u}$, indicating that the factor of amplification is also constant regardless of variations in $a_{3}^{u}$. The last step is to examine how $\left(\mathbf{B}^{\mathrm{NU}}\right)_{\text {MAX }}$ and $\mathbf{B}_{\mathrm{U}}$ depend on the period of the Rayleigh wave (Fig. 3(c)). This test is important because seismic waves generally consist of various components with different frequencies. For periods longer than $20 \mathrm{~s}$, the factor of amplification is largely constant, similar to the results obtained when $a_{3}^{l}$ and $a_{3}^{u}$ are varied. However, for periods shorter than $15 \mathrm{~s}$, the factor of amplification is smaller than that for longer periods. Given that the dominant periods of Rayleigh waves are longer than $15 \mathrm{~s}$, this change in amplification at a period of about $15 \mathrm{~s}$ can be ignored in the present study. Taking the above results into account, enhancement of the piezomagnetic effect is expected to be observed near the edges of the magnetization boundary, regardless of the depth and thickness of the magnetized body and of the wavenumber of the Rayleigh wave.

\section{Discussion}

The numerical estimations outlined in the preceding section focus on a persistent seismic wave with a single frequency as a source of the piezomagnetic effect: temporal variations in seismic waves are ignored. In contrast, the propagation of actual seismic waves is a transient phenomenon. To provide a precise description of the piezomagnetic field that accompanies transient seismic waves, it is necessary to consider the explicit forms of seismic waves and calculate the inverse Fourier transform of the formula obtained in the present study. For this exact estimation, this treatment must take place at the initial moment at which seismic waves cross the magnetization boundary or the observation site. However, the focus here is on the time during which the seismic wave crosses the boundary or observation site, without considering the initial moment. In this case, the calculation of the piezomagnetic field for a persistent elastic wave provides a good approximation of the field for a transient elastic wave (Yamazaki, 2010).

It has been confirmed that the piezomagnetic field is significantly enhanced near the edges of the magnetization boundary. Moreover, the obtained amplitudes of the signal appear to be of detectable magnitude. Nevertheless, the above results do not indicate that the piezomagnetic effect is always a plausible mechanism in terms of generating coseismic variations in the magnetic field. The magnitude of piezomagnetic signals depends on the intensity and spatial structure of the initial magnetization, and the amplitudes of the expected piezomagnetic signals are obtained only for assumed values. In addition, the formulation proposed in this paper assumes that the Earth's crust is electrically resistive. 
Yamazaki (2010) derived an analytical expression of the piezomagnetic field that accompanies seismic waves in a crust with finite conductivity and uniform magnetization and reported that conductivity may sometimes induce a significant increase or decrease in the time-varying piezomagnetic signals. The result obtained here for non-uniformly magnetized crust may vary in the case of relatively high conductivity. In this sense, the results outlined in the preceding section represent a prediction corresponding to a specific set of parameters; however, given that the assumed parameters are not unrealistic, the results can be considered to be realistic for selected situations. The assumed intensity of initial magnetization (i.e., $10 \mathrm{~A} / \mathrm{m}$ ) is relatively large, but has previously been estimated as the initial magnetization of the Earth's crust (e.g. Nishida et al., 2004). The assumed amplitudes of the Rayleigh wave are also rather large, but amplitudes of this magnitude are observed at farfield sites in the case of large events, such as the 2004 Sumatra-Andaman earthquake and the 2010 Chile earthquake. Therefore, it is concluded that the piezomagnetic effect may be a plausible mechanism of co-seismic variations in the geomagnetic field in certain settings, particularly if the magnetometer is installed near magnetization boundaries.

\section{Conclusion}

In a wavenumber space, the piezomagnetic field arising from the propagation of seismic plane waves over a blockshaped magnetization has an analytically closed-form expression. Spatial variations in real-space are rapidly calculated after applying a reverse Fourier transform.

Examples of numerical calculations performed using the developed formulae reveal that the piezomagnetic signals are considerably enhanced near the magnetization boundary. Enhancements are commonly seen, regardless of changes in the depth and thickness of the magnetized layer. This finding suggests that if the observation site is located near the magnetization boundary, the piezomagnetic effect is a plausible mechanism in explaining variations in the geomagnetic field during the propagation of seismic Rayleigh waves, along with other mechanisms, such as the electrokinetic effect and the induction effect due to ground motions.

Acknowledgments. The author thanks Dr. Koki Aizawa (Earthquake Research Institute, University of Tokyo), Mr. Kento Taira (Graduate School of Science, Kyoto University), and Dr. Toshihiko Iyemori (World Data Center for Geomagnetism, Kyoto University) for discussions that motivated this study. Comments provided by two reviewers, Dr. Gilda Currenti and Dr. Takeshi Hashimoto, greatly improved the quality of the manuscript, as did the patient editorial handling of the manuscript by the Associate Editor, Dr. Kiyoshi Yomogida. Figures 2 and 3 were drawn using Generic Mapping Tools (Wessel and Smith, 1998).

\section{References}

Abdul Azeez, K. K., C. Manoj, K. Veeraswamy, and T. Harinarayana, Coseismic EM signals in magnetotelluric measurement-a case study during Bhuj earthquake (26th January 2001), India, Earth Planets Space, 61, 973-981, 2009.

Arfken, G. B. and H. J. Weber, Mathematical Methods for Physics, 5th ed., 1112 pp., Academic Press, San Diego, 2000.

Bordes, C., L. Jouniaux, S. Garambois, M. Dietrich, J. P. Pozzi, and S. Gaffet, Evidence of the theoretically predicted seismo-magnetic con- version, Geophys. J. Int., 174, 489-504, 2008.

Eleman, F., The response of magnetic instrument to earthquake waves, $J$. Geomag. Geoelectr., 18, 43-72, 1965.

Garambois, S. and M. Dietrich, Seismoelectric wave conversion in porous media: field measurements and transfer function analysis, Geophysics, 66, 1417-1430, 2001.

Honkura, Y., M. Matsushima, N. Oshiman, M. K. Tunçer, Ş. Bariş, A. Ito, Y. Iio, and A. M. Işikara, Small electric and magnetic signals observed before the arrival of seismic wave, Earth Planets Space, 54, e9-e12, 2002.

Honkura, Y., H. Satoh, and N. Ujihara, Seismic dynamo effects associated with the M7.0 earthquake of 26 May 2003 off Miyagi Prefecture and the M6.2 earthquake in northern Miyagi Prefecture, NE Japan, Earth Planets Space, 56, 109-114, 2004.

Honkura, Y., Y. Ogawa, M. Matsushima, S. Nagaoka, N. Ujihara, and T. Yamawaki, A model for observed circular polarized electric fields coincident with the passage of large seismic waves, J. Geophys. Res., 114, B10103, doi:10.1029/2008JB006117, 2009.

Ishido, T. and H. Mizutani, Experimental and theoretical basis of electrokinetic phenomena in rock-water systems and its application to geophysics, J. Geophys. Res., 86, 1763-1775, 1981.

Iyemori, T., T. Kamei, Y. Tanaka, M. Takeda, T. Hashimoto, T. Araki, T. Okamoto, K. Watanabe, N. Sumitomo, and N. Oshiman, Co-seismic geomagnetic variations observed at the 1995 Hyogoken-nanbu earthquake, J. Geomag. Geoelectr., 48, 1059-1070, 1996.

Iyemori, T., M. Nosé, D. Han, Y. Gao, M. Hashizume, N. Choosakul, H. Shinagawa, Y. Tanaka, M. Utsugi, A. Saito, H. McCreadie, Y. Odagi, and F. Yang, Geomagnetic pulsations caused by the Sumatra earthquake on December 26, 2004, Geophys. Res. Lett., 32, L20807, doi:10.1029/2005GL024083, 2005.

Lay, T. and T. C. Wallace, Modern Global Seismology, 521 pp., Academic Press, San Diego, 1995.

Matsushima, M., Y. Honkura, N. Oshiman, S. Baris, M. K. Tuncer, S. B. Tank, C. Celik, F. Takahashi, M. Nakanishi, R. Yoshimura, R. Pektas, T. Komut, A. Ito, Y. Iio, and A. M. Isikara, Seismo-electromagnetic effect associated with the Izmit Earthquake and its aftershock, Bull. Seismol. Soc. Am., 92, 350-360, 2002.

Nagata, T., Basic magnetic properties of rocks under the effect of mechanical stresses, Tectonophysics, 9, 167-195, 1970.

Nishida, Y., Y. Sugisaki, K. Takahashi, M. Utsugi, and H. Oshima, Tectonomagnetic study in the eastern part of Hokkaido, NE Japan: Discrepancy between observed and calculated results, Earth Planets Space, 56, 1049-1058, 2004.

Nishida, Y., M. Utsugi, and T. Mogi, Tectonomagnetic study in the eastern part of Hokkaido, NE Japan (II): Magnetic fields related with the 2003 Tokachi-oki earthquake and the 2004 Kushiro-oki earthquake, Earth Planets Space, 59, 1181-1186, 2007.

Ogawa, T. and H. Utada, Electromagnetic signals related to incidence of a teleseismic body wave into a subsurface piezoelectric body, Earth Planets Space, 52, 253-260, 2000a.

Ogawa, T. and H. Utada, Coseismic piezoelectric effects due to a dislocation 1. An analytic far and early-time field solution in a homogeneous whole space, Phys. Earth Planet. Inter., 121, 273-288, 2000b.

Okubo, K., A. Takeuchi, Y. Suyama, M. Utsugi, Y. Sasai, Y. Nakamura, and N. Takeuchi, Observation of magnetic signals from earthquake faulting: co-faulting signals in the Iwate-Miyagi Nairiku Earthquake of M 7.2, NE Japan, Eos Trans. AGU, 90(52), Fall Meet. Suppl., Abstract NH31C1125, 2009.

Oshiman, N., Enhancement of tectonomagnetic change due to non-uniform magnetization in the Earth's crust-two dimensional case studies, $J$. Geomag. Geoelectr., 42, 607-619, 1990.

Pride, S., Governing equation for the coupled electromagnetics and acoustics of porous media, Phys. Rev., B50, 15678-15696, 1994.

Sasai, Y., Application of the elasticity theory of dislocations to tectonomagnetic modelling, Bull. Earthq. Res. Inst., Univ. Tokyo, 54, 1-29, 1980.

Sasai, Y., Tectonomagnetic modeling on the basis of the linear piezomagnetic effect, Bull. Earthq. Res. Inst., Univ. Tokyo, 66, 587-722, 1991.

Sasai, Y., Piezomagnetic fields produced by dislocation sources, Surv. Geophys., 15, 363-382, 1994.

Stacey, F. D., The seismomagnetic effect, Pure Appl. Geophys., 58, 5-22, 1964.

Stacey, F. D. and M. J. S. Johnston, Theory of the piezomagnetic effect in titanomagnetite-bearing rocks, Pure Appl. Geophys., 97, 146-155, 1972.

Taira, K., T. Iyemori, and D. Han, Geomagnetic variations observed at 
the arrival of seismic wave of Sumatra Earthquake, Society of Geomagnetism and Earth, Planetary and Space Sciences (SGEPSS), 2009 Fall meeting, abstract, A003-02, 2009.

Ujihara, N., Y. Honkura, and Y. Ogawa, Electric and magnetic field variations arising from the seismic dynamo effect for aftershocks of the M7.0 earthquake of 26 May 2003 off Miyagi Prefecture, NE Japan, Earth Planets Space, 56, 115-124, 2004.

Yamazaki, K., Calculation of the piezomagnetic field arising from uniform regional stress in inhomogeneously magnetized crust, Earth Planets Space, 61, 1163-1168, 2009.
Yamazaki, K., Piezomagnetic fields arising from the propagation of teleseismic waves in magnetized crust with finite conductivity, Geophys. J. Int., 2010 (in press).

Wessel, P. and W. H. Smith, New, improved version of generic mapping tools released, Eos Trans. AGU, 79(47), 579, 1998.

K. Yamazaki (e-mail: kenichi@rcep.dpri.kyoto-u.ac.jp) 